Sonderdruck aus der

Zeitschrift für Pflanzenernährung und Bodenkunde

116. Band, Hefi 2, 1967, Seite 89-96

(c) Verlag Chemie, GmbH., Weinheim/Bergstr.

\title{
Fraktionierung von Huminstoffen eines Podsols durch Dialyse
}

\author{
Von $F$. Martin-Martinez
}

Centro de Edafologia y Biologia Aplicada des Quarto, Sevilla

(Direktor: Professor F. Gonzales Garcia)

(Eingegangen: 8. 3. 1965)

\section{Einleitung}

Nach der klassischen Einteilung werden bei den Huminstoffen die säurelöslichen Fulvo- und die säureunlöslichen Huminsäuren unterschieden. Je länger man sich mit der Untersuchung dieser Stoffgruppen beschäftigte, desto mehr stellt man fest, daß ein kontinuierlicher Übergang zwischen diesen beiden Gruppen besteht.

Zur Untersuchung der Huminstofte und des Humifizierungsprozesses sind verschiedene Fraktionierungsmethoden angewandt worden. Kyuma (4) führte fraktionierte Fällungen der Huminsäuren in basischer Lösung mit Alkohol aus. Er steigerte diese Konzentration von 12,5 bis $80 \%$ (v/v) durch Zugabe von abs. Athanol zu der alkalischen Lösung.

Salfeld (8) fraktionierte Huminsäuren eines Tschernosems durch aufeinanderfolgende Extraktion mit wasserhaltigem Tetrahydrofuran mit einem Wassergehalt von 5, 10 und 20\%. Sowden (9) fraktionierte Fulvosäuren eines Podsols durch Zugabe von steigenden Mengen an $\mathrm{Pb}^{++}, \mathrm{Ba}^{++}, \mathrm{Cu}^{++}$-Ionen und untersuchte die erhaltenen Fraktionen auf ihre Eigenschaften als Carbonsäuren und Phenole. Martin (6) fraktionierte die Fulvosäuren eines Podsols, indem er deren unterschiedliche Löslichkeit in Tetrahydrofuran ausnützte; er untersuchte die Prozesse der Humifizierung mittels Bestimmung der funktionellen Gruppen der erhaltenen Fraktionen.

Verschiedene chromatographische Methoden sind zur Fraktionierung der Huminsäuren und ebenso zu der der Fulvosäuren angewandt worden.

Weitere Fraktionierungen sind durch die Verteilung des Molekulargewichtes mittels Ultrazentrifuge und durch Gelfiltration mit Sephadex $(1,7)$ vorgenommen worden.

In der vorliegenden Untersuchung wird die Fraktionierung der Huminstoffe eines Podsols durch Dialyse bearbeitet, wobei eine nähere Charakterisierung der Humin- und Fulvosäurenfraktionen durchgeführt wird. 


\section{Experimenteller Teil}

Material: Für die Versuche wurde der B-Horizont eines Podsols (Vollbüttel b. Braunschweig, Deutschland) benutzt. Der Boden wurde sorgfältig zerkleinert und schließlich durch ein $1 \mathrm{~mm}$ Sieb gesiebt. Extraktion der Fulvosäuren: $1 \mathrm{~kg}$ der Bodenprobe wurde mit 311 n-Salzsäure 3 Stunden geschüttelt. Nach Zugabe von Kaliumbisulfat (um die Tonsubstanz auszufällen) und Dekantieren wurde bei $3000 \mathrm{rpm}$ zentrifugiert. Mit $5 \mathrm{n}$-Natronlauge wurde die Lösung auf $\mathrm{pH} 4$ gebracht und dadurch die Fulvosäuren ausgefällt. Nach nochmaliger Zentrifugation wurde der Niederschlag in $0,1 \mathrm{n}-\mathrm{NaOH}$ gelöst und bei $17000 \mathrm{rpm} 30$ Minuten wiederum zentrifugiert. Die überstehende Lösung wurde mit Salzsäure bis zu einem pH-Wert von 4 angesäuert und der ausgefallene Niederschlag bis zur $\mathrm{Cl}^{-}$-Freiheit gegen Wasser dialysiert. Die im Dialysator verbleibende dunkelbraune Lösung wurde mit Dowex 50 (H-Form) geschüttelt, von dem Umtauscher abgetrennt und durch eine Kolonne mit Dowex 2 (OH-Form) perkoliert. Von dem Perkolat wurde im Vakuum das Wasser abdestilliert und der Rückstand anschließend im Hochvakuum getrocknet.

Extraktion der Huminsäuren: $1 \mathrm{~kg}$ der Bodenprobe wurde mit 31 $0,1 \mathrm{n}-\mathrm{NaOH}$ geschüttelt und die Lösung nach Zugabe von Kaliumbisulfat zentrifugiert. Anschließend wurden nach Ansäuern mit Salzsäure auf einen pH-Wert von 1 die Huminsäuren ausgefällt. Diese wurden dann 4- bis $5 \mathrm{mal}$ in Natronlauge gelöst und mit Säure gefällt. Der Niederschlag wird schließlich wieder in $0,1 \mathrm{n}$-Natronlauge gelöst und bei $17000 \mathrm{rpm}$ zentrifugiert. Nach Ansäuern mit Salzsäure auf einen $\mathrm{pH}$-Wert von 1 wurde bis zur $\mathrm{Cl}^{-}$-Freiheit gegen destilliertes Wasser dialysiert. Von dem Dialyserückstand wurde das Wasser abdestilliert und die zurückbleibenden Huminsäuren im Hochvakuum getrocknet.

\section{Methoden}

$5 \mathrm{~g}$ Fulvosäuren werden in $150 \mathrm{ml}$ dest. Wasser gelöst und in einem Schlauch gegen dest. Wasser dialysiert. Alle 48 Std. wird die Außenlösung (Dialysat) gesammelt und im Vakuum eingedampet. Insgesamt wurden 5 Fraktionen abgenommen.

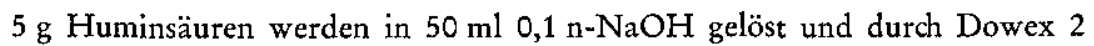
(OH-Form) und durch Dowex 50 (H-Form) perkoliert. Das Perkolat wurde auf $100 \mathrm{ml}$ gebracht und in einem Dialysierschlauch gegen $0,2 \mathrm{n}-\mathrm{NaOH}$ dialy siert. Das Dialysat wurde alle 48 Stunden gesammelt und durch Dowex 2 und Dowex 50 perkoliert. Das Eluat ist im Vakuum zur Trockne eingedampft worden. Auch in diesem Falle sind 5 Fraktionen gesammelt worden.

Von den verschiedenen Fraktionen sind $1 \mathrm{mg}$ der Probe und $200 \mathrm{mg} \mathrm{KBr}$ in einer Vibrationsmühle gemischt, eine Stunde bei $110^{\circ} \mathrm{C}$ getrocknet und dann zu einer Tablette gepreßt worden. Die Spektren wurden gegen eine gleich- 
behandelte KBr-Tablette mit einem Perkin Elmer Infracord 137 aufgenommen.

Die Fulvosäuren wurden potentiometrisch titriert. Dazu wurden $50 \mathrm{mg}$ in $75 \mathrm{ml}$ Wasser gelöst und mit $0,02 \mathrm{n}-\mathrm{NaOH}$ in einem Beckman Mod. 8509 Potentiometer titriert.

Bei den Huminsäuren wurde ebenfalls eine potentiometrische Titration durchgeführt. $50 \mathrm{mg}$ Huminsäuren wurden in $25 \mathrm{ml} 0,1 \mathrm{n}-\mathrm{NaOH}$ gelöst und nach Perkolieren durch Dowex 2 und Dowex 50 auf ein Volumen von $75 \mathrm{ml}$ gebracht.

\section{Ergebnisse und Diskussion}

Die IR-Spektren der ursprünglichen Fulvosäure und der 5 Fraktionen sind in Abbildung 1 wiedergegeben.

Das Spektrum der ursprünglichen Fulvosäure (AF) zeigt die üblichen Banden bei $3420,1720,1610$ und $1250 \mathrm{~cm}^{-1}$. Diese Banden werden allgemein den $\mathrm{OH}-$, $\mathrm{C}=\mathrm{O}-, \mathrm{C}=\mathrm{C}$ - und $\mathrm{C}-\mathrm{O}-\mathrm{Sch}$ wingungen zugeordnet. Alle diese Banden sind auch in den Spektren der dialysierten Fraktionen festzustellen. Die Höhe der

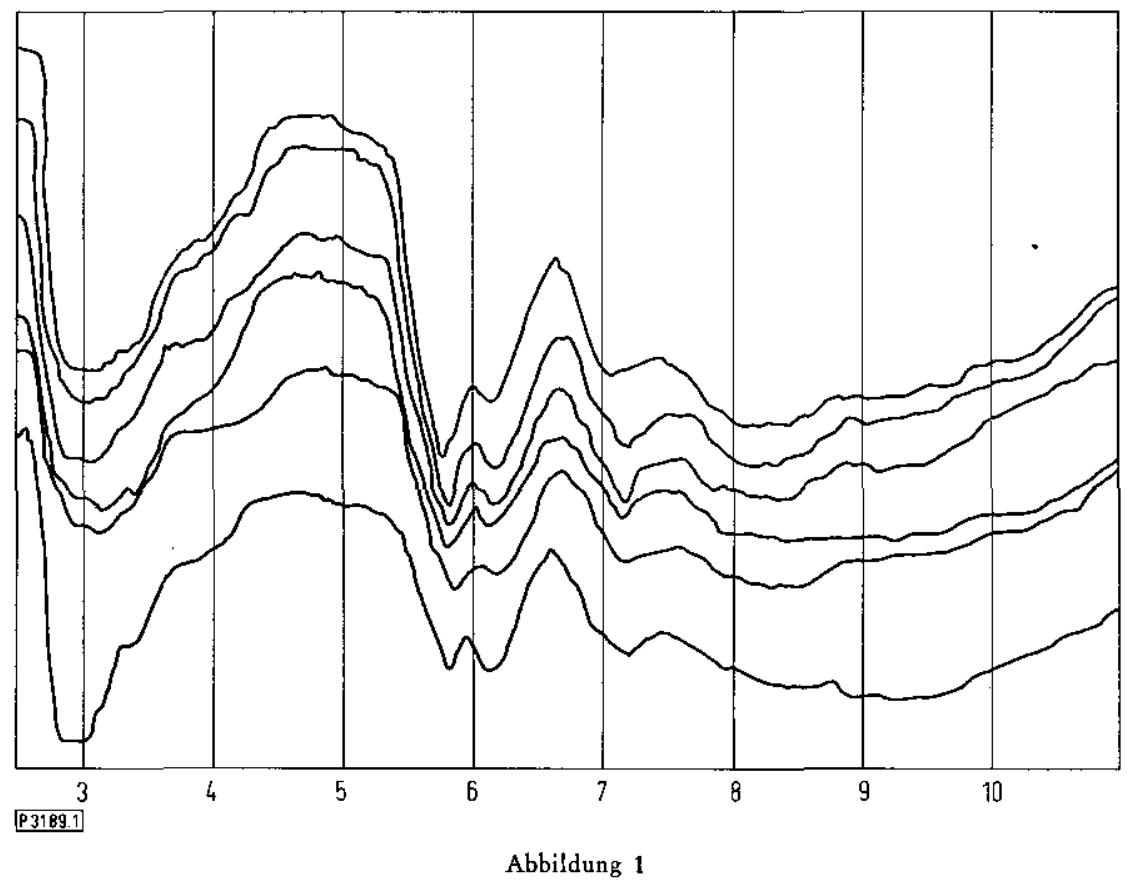

IR-Spektren der ursprïnglichen Fulvosäure und der 5 Fraktionen IR-spektra of the original fulvic acid and the 5 fractions 
Banden der $\mathrm{C}=\mathrm{O}-\mathrm{Gruppen}$ bei $1720 \mathrm{~cm}^{-1}$ der Fraktionen nimmt $\mathrm{ab}$, während die Höhe der anderen Banden gleichgeblieben ist.

Der Gehalt an Carboxylgruppen nimmt von der ersten bis zur fünften Fraktion $a b$, wenn man die Bande bei $1720 \mathrm{~cm}^{-1}$ Carboxylgruppen zuordnet. Damit kann ein Zusammenhang zwischen dem Gehalt an Carboxylgruppen und der Dialysiergeschwindigkeit festgestellt werden. Der Unterschied der Dialysiergeschwindigkeit kann möglicherweise auch auf einer zunehmenden Größe der Teilchen beruhen. Wenn dieses zutriff, haben die niedermolekularen Anteile von den Fulvosäuren einen höheren Gehalt an Carboxylgruppen.

In Abbildung 2 sind die Neutralisationskurven der verschiedenen Fraktionen angegeben.

Es besteht eine gute Korrelation zwischen dem Gehalt an Carboxylgruppen, die aus den IR-Spektren hervorgehen, und der Neutralisationskapazität.

Die Abbildung 3 zeigt die IR-Spektren der fünf Fraktionen und der ursprünglichen Huminsäuren.

Wie im Falle der Fulvosäuren beobachtet man auch bei den Huminsäurefraktionen eine Abnahme der Carboxylgruppen, da die Höhe der entsprechenden Bande mit der Dauer der Dialyse fortlaufend abnimmt.

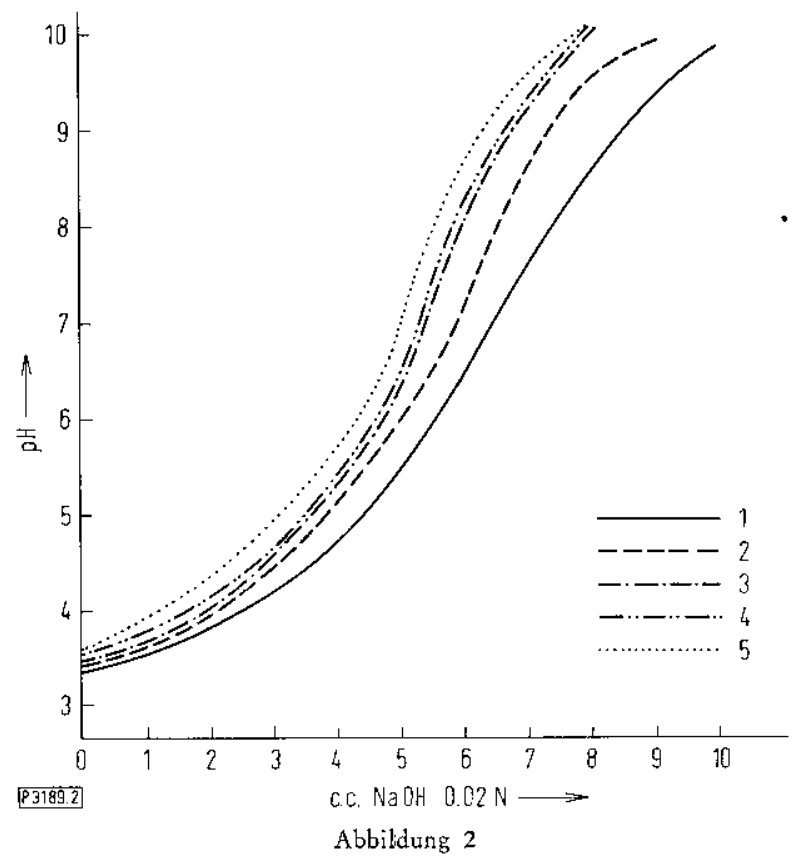

Neutralisationskurven der ursprünglichen Fulvosäurc und der 5 Fraktionen

Neutralization curves of the original fulvic acid and of the 5 fractions 


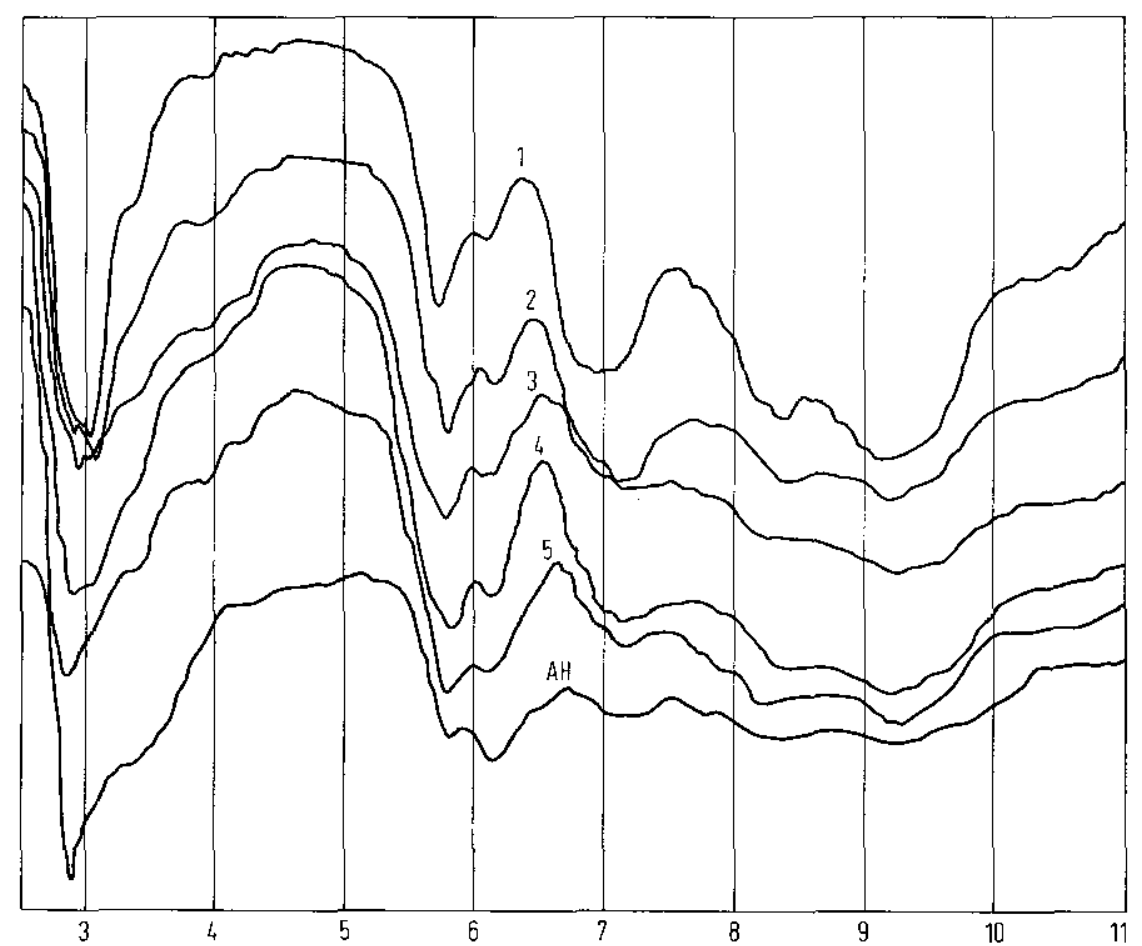

P3189.3

Abbildung 3

IR-Spektren der ursprünglichen Huminsäurenfraktion (AH) und der durch Dialyse erhaltenen 5 Fraktionen

IR-spectra of the original humic acid fraction $(\mathrm{AH})$ and of the 5 fractions separated by dialysis

Die Kurven der potentiometrischen Titration der Huminsäurenfraktionen sind in Abbildung 4 angegeben.

Im Gegensatz zu den Fulvosäuren nimmt die Neutralisationskapazität und das Säureäquivalent mit fortschreitender Dialyse zu. Kumada (5) findet ebenfalls mit fortschreitender Humifizierung eine Zunahme der Neutralisationskapazität.

Die Neutralisationskapazität verläuft mit dem Säurcäquivalent parallel. Die Acidität der Huminsäuren kann durch Carboxyl- und Hydroxyl-Gruppen verursacht werden. Bei einer näheren Betrachtung der Kurven der potentiometrischen Bestimmungen (Abb. 2 und 4) stellt man fest, daß die Kurven der Fulvosäuren nur einen Wendepunkt besitzen. Dagegen weisen die Kurven der Huminsäuren, mit Ausnahme der ersten Fraktion, zwei Wendepunkte auf, die durch die Neutralisation von stärker oder schwächer sauren Gruppen, wie 


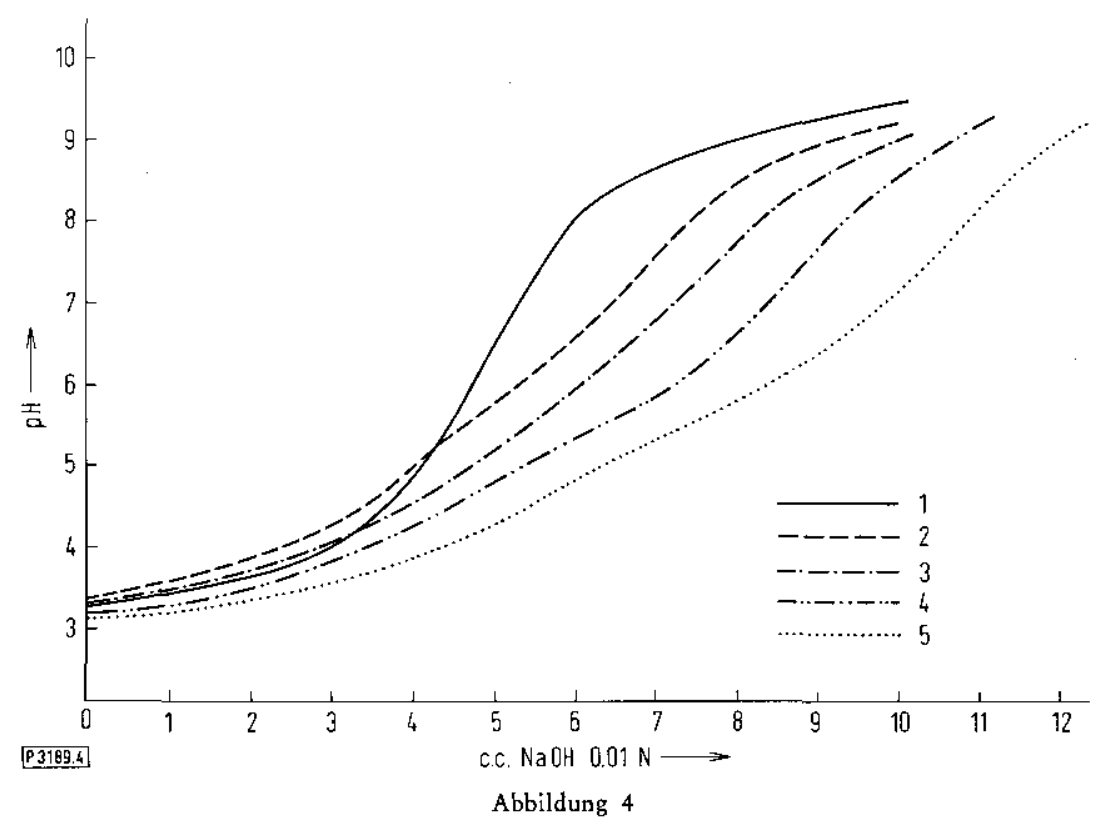

Neutralisationskurven der ursprünglichen Huminsäurenfraktion und der durch Dialyse abgetrennten 5 Fraktionen

Neutralization curves of the original humic acid fraction and the 5 fractions separated by dialysis

Carboxyl- und phenolischen Hydroxylgruppen, verursacht werden können. So sind auch in den Infrarotspektren (Abb. 1 und 3) die Banden der Hydroxylgruppen bei $3420 \mathrm{~cm}^{-1}$ bei den Huminsäuren ausgeprägter als bei den Fulvosäuren.

In Übereinstimmung mit diesen Ergebnissen erfolgt bei der Reduktion der Carboxylgruppen in Fulvosäuren eine erhebliche Abnahme der Neutralisationskapazität (6). Bei den Huminsäuren konnte keine vollständige Reduktion erzielt werden. Bei der Reduktion verschiebt sich hier das Verhältnis der Sauerstofffunktionen zu Gunsten der OH-Gruppen.

Geht man von der mehrfach geäußerten Vorstellung aus, daß im Verlaufe der Bildung der Huminstoffe die höhermolekularen Anteile sich durch Kondensationsreaktionen aus niedermolekularen Verbindungen aufbauen, so ist der Prozeß mit dem Verlust an Carboxyl- und der Zunahme an Hydroxylgruppen verbunden. Dabei setzt man voraus, daß die Fulvosäuren niedermolekulare Anteile einer polymerhomologen Reihe der Huminstoffe und der Huminsäuren deren höhermolekulare Glieder sind.

Diese Vorstellungen sind in Übereinstimmung mit einem schematischen Modell von Larina und Kasatochkin (2), die den Aufbau dieser Fraktionen aus 
einem aromatischen Kern mit äußeren Seitenketten, an denen sich die funktionellen Gruppen, wie Carboxyl- und Hydroxylgruppen befinden, beschrieben.

Nach Kononowa (3) gilt diese Struktur für beide Fraktionen. In den Fulvosäuren herrschen die Seitenketten über die Kerne, in den Huminsäuren dagegen, die Kerne über die Seitenketten vor. Je nachdem, ob die Hydroxylgruppen an der Seitenkette oder im Kern vorhanden sind, erhöhen diese funktionellen Gruppen die Acidität der Huminsäuren.

\section{Zusammenfassung}

Die Fulvosäuren und Huminsäuren eines Podsols werden durch Dialyse in 5 Fraktionen getrennt. Es werden IR-Spektren und die Neutralisationskurven jeder der isolierten Fraktionen gemessen und verglichen. Aus den Ergebnissen wird geschlossen, daß in den Fulvosäurenfraktionen die Acidität hauptsächlich durch Carboxylgruppen verursacht wird, deren Anzahl in Fraktionen, die durch Dialyse gewonnen werden, mit fortschreitender Zeitdauer abnimmt. Bei den Fraktionen der Huminsäuren findet mit Fortschreiten der Dialyse ebenfalls eine Abnahme der Carboxylgruppen statt, während jedoch die Neutralisationskapazität zunimmt. Dies bedeutet, daß die Acidität zunehmend durch phenolische Hydroxylgruppen verursacht wird. Unter Zugrundelegung gewisser Modellvorstellungen ist somit das Fortschreiten der Humifizierung mit einer Abnahme des Gehaltes an Carboxyl- und einer Zunahme an Hydroxylgruppen verbunden.

1. Ferrari, G. and Dell'Agnola, G.: Fractionation of the organic matter of soil by gel filtration through Sephadex. Soil Sci. 96, 418-421 (1963).

2. Larina, N. K. und Kasatodhkin, V. l.: Pochvovedenie 9, 28 (1957).

3. Kononowa, M. M.: Soil Organic Matter. Pergamon Press (1961).

4. Kyuma, K.: A fractional precipitation technique applied to soil humic substances. Soil Sci. and Plant Nutrition 10, 33-35 (1964).

5. Kumada, K.: Soil and Plant Food. 1, 29, 75 (1955); 2, 44 (1956); 3, 152 (1958).

6. Martin, F., Dubach, P., Metba, N. C. und Desel, H.: Z. Pflanzenernähr., Düng., Bodenkunde 103, 27-39 (1963).

7. Mehta, N. C., Dubach, P. und Deuel, H.: Untersuchungen der Molekulargewichtsverteilung von Huminstoffen durch Gelfiltration an Sephadex. Z. Pflanzenernähr., Düng., Bodenkunde 102, 128-137 (1963).

8. Salfeld, J. Chr.: Landbauforschung Völkenrode 14, 131-136 (1964).

9. Sowden, F. J. and Deuel, H.: Fractionation of fulvic acids from the B-horizont of a podzol. Soil Sci. 91, 44-48 (1961).

\section{Fractionation of Humic Substances of a Podzol by Dialysis}

by F. Martin-Martinez

The fulvic and humic acids of a podzol were fractionated into five fractions by means of dialysis. In each fraction the process of humification was studied through 
IR-spectra and the curve of neutralization. In the fractions of the fulvic acids, the acidity is due to the carboxyl groups and with the progress of humification one observes a decrease of these groups. On the contrary, in the fractions of the humic acids one observes a decrease in the carboxyl groups with the progress of humification but the neutralization capacity increases. This means that the acidity is caused in the humic acids by the phenolic $\mathrm{OH}$ groups. In both cases the humification progresses with a loss of carboxyl groups and a gain of $\mathrm{OH}$ groups. 